\title{
Comparison of Polarimetric Decomposition Techniques to Suppress Subsurface Clutter in GPR Applications
}

\author{
Sebastian G. Wirth and Ivor L. Morrow \\ Cranfield University, Laboratory of Electromagnetic Systems Engineering, \\ Shrivenham Campus, Oxfordshire, SN6 8LA, UK. \\ Email: sebastian.wirth@cranfield.ac.uk, i.l.morrow@cranfield.ac.uk
}

\begin{abstract}
The effect of different decomposition techniques on the imaging and detection accuracy for polarimetric surface penetrating radar data is studied. We derive the general expressions for coherent polarimetric decomposition using the model based polarimetric decomposition of Yamaguchi technique and compare these with some Stokes and Pauli coherent polarisation decomposition parameters. These mathematical treatments are then applied to laboratory based X-band (8.2$12.4 \mathrm{GHz})$ full polarimetry near-field radar measurements taken of shallow buried reference and calibration objects and different landmine types. The Yamaguchi polarimetry filters demonstated significant surface and sub-surface clutter reduction and contrast in subsurface imagery, with some loss in signal power. The Stokes and Pauli parameters demonstrated similar clutter reduction in subsurface imagery providing additional beneficial information on the targets scattering mechanism. Combining these techniques contributes to an improvement of subsurface radar discrimination and understanding of the target type.
\end{abstract}

\section{Introduction}

Ground penetrating radar (GPR) is increasingly being used for landmine detection [1],[2]. In contrast to metal detecting, GPR can be used for detection of low-metal content or non-metal content targets. Subsurface radar suffers from two typical problems, a strong clutter response from the surface and high signal-to-clutter power levels in the underground [3],[4]. This paper investigates clutter reduction by experimental study of two polarimetric decomposition techniques; the model based technique of Yamaguchi [5] and Pauli [6] polarisation parameter analysis.

A series of laboratory measurements were conducted using a full-polarimetry X-band (8.2-12.4GHz) GPR system. The GPR system is operated in the near-field with a small, but finite, bistatic angle and therefore the data collected can be considered bistatic. Monostatic calibration of wideband GPR systems where the scattering matrix for a reciprocal target remains symmetric are well developed [7]. In recent years interest in bistatic RCS measurements [8] and bistatic/multistatic GPR systems has increased due to the perception that the obtained results give more information on complex object shape and material composition. Reduced order bistatic calibration procedures have been reported in the literature for RCS measurement [9] and for GPR [10] operation. The fully polarimetric (FP) calibration technique is described which involves initally acquiring the mutual coupling or isolation coefficients over a narrow range of bistatic angles on the polarimetric antenna array and extrapolating these to an equivalent monostatic array configuration.

The Yamaguchi clutter suppression technique synthesises a complex null polarisation state that minimises targeted clutter scattering pixels. Using the null polarisation state matrix it is possible to repeatly apply this across the B-scan and produce

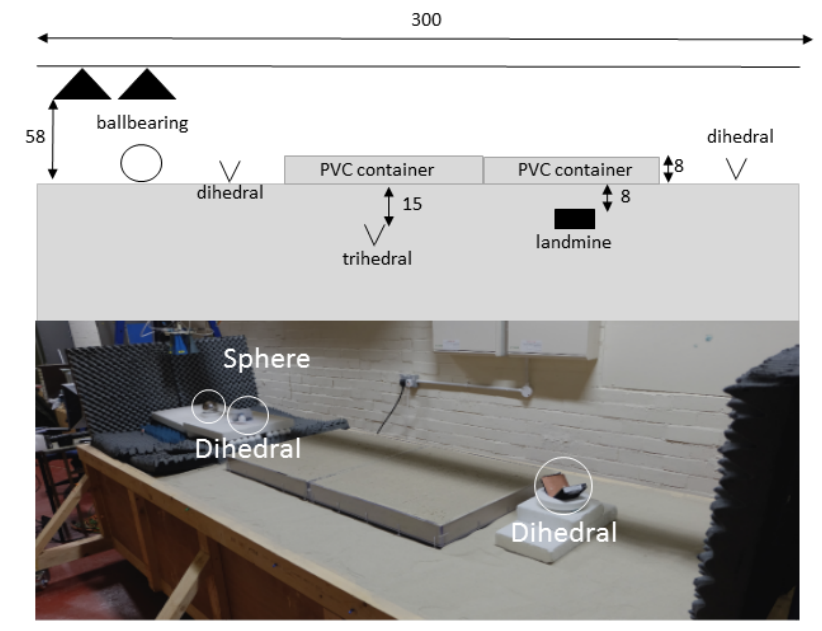

Figure 1. Indoor laboratory experiment setup: Top view depicts a cross-sectional schematic diagram showing the GPR geometry and location of the reference and buried objects (all units are in $\mathrm{cms}$ ) and the Bottom view shows the measurement environment.

polarimetric co- and cross-polar power responses with reduced image clutter. The results are also compared with other polarimetric imagery products calculated from the data using Pauli decompositions $a, b, c$ and Stokes parameter $I_{o}$.

This paper is organised as follows; Section 2. describes the laboratory experiment methodology, radar waveform and antenna array setup. Section 3. outlines the calibration routine and bistatic correction factor applied to reformat the data as monostatic measurements. The mathematical expressions for polarimetric filtering are given in Section 4. and Section 5. describes the Stokes and Pauli polarimetric decompositions that can also be applied to discriminate the target scattering mechanism. Section 6. discusses the performance of the various techniques and provides summary conclusions and an 


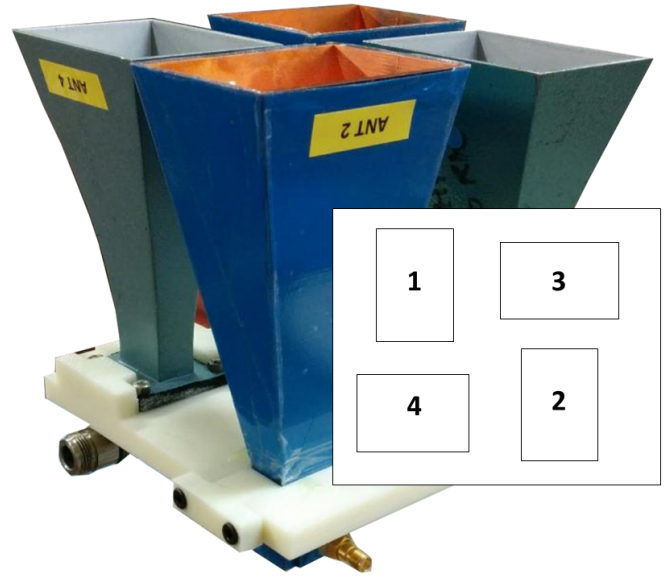

Figure 2. The X-Band fully polarimetric Pyramidal horn antenna array. The antenna array consists of two pairs of horizontally and vertically polarised antennas to achieve polarisation diversity (the inset diagram depicts the orientation of the rectangular apertures).

engineering appraisal.

\section{Measurement System and Methodology}

A stepped frequency continuous wave (SFCW) is implemented using a Rhode \& Schwarz ZVA 10675 E vector network analyser as the coherent transmitter-receiver unit. The other parts of the system are the transmitting and the receiving antenna array and the computer to automate position control, data acquisation, signal processing and electromagnetic visualisation. The experimental set-up is shown in Figure 1.

\subsection{Experimental Setup}

Experiments have been conducted on the indoor soil facility, shown in Figure 1, which consists of one soil bay (2.4Lx1.2Wx0.8H meters) filled with sand and an adjacent bay (1.2Lx1.2Wx0.8H meters) lined with RAM on which calibration targets can be placed. The soil is maintained within a 3-8\% moisture content and at a constant room temperature of $25^{\circ} \mathrm{C}$. A Near-Field Measurement System (NFMS) has been erected over the two bays with a $3.0 \mathrm{~m}$ long linear automated positioner. The antenna array shown in Figure 2 implements two pairs of horizontally and vertically polarised antennas to achieve polarisation diversity and the figure inset depicts the antenna aperture orientation. The radar system is configured to sequentially measure the scattering parameter $S_{21}$ across the X-band $(8.2-12.4 \mathrm{GHz})$ frequency spectrum in all possible combinations available from the $\mathrm{H}$ - and $\mathrm{V}$-antennas, i.e. $\mathrm{H}$-transmitting and $\mathrm{H}$-receiving and soforth). Then the FP scattering matrix $(\mathrm{HH}, \mathrm{HV}, \mathrm{VH}, \mathrm{HH})$ for a resolution element may be determined. The array is mounted $58 \mathrm{cms}$ above the soil surface and acquires the scattering matrix in $1.0 \mathrm{~cm}$ measurement intervals. The measured $S_{11}$ response for all the antennas in the array is $\leq-12 \mathrm{~dB}$ and the measured co- and cross-polar mutual coupling in E- and H-planes is $\leq-35 \mathrm{~dB}$ and $\leq-60 \mathrm{~dB}$, respectively.

As previously mentioned in one soil bay several reference targets were placed above the surface of the sand resting on a $4 \mathrm{~cm}$ thick block of polystyrene foam and a bed of radar absorbent material (RAM). The foam layer permits more precise localisation of the reference targets scattered response. These included a $4 \mathrm{~cm}$ metal sphere and two $7 \mathrm{~cm}$ square sided dihedral reflectors with their seam rotated at an $22.5^{\circ}$ angle to the direction of antenna movement. In a second region two (70Lx70Wx9D cms) Perspex trays were laid ontop of the sand and completely filled with sand. Under one Perspex tray another trihedral, $7 \mathrm{~cm}$ square sided, was buried at a depth of $23 \mathrm{~cm}$ from the surface of the filled tray. Under the second Perspex tray a TS-50 landmine diameter $6 \mathrm{~cm}$ and thickness $4 \mathrm{~cm}$ was buried at a depth of $16 \mathrm{~cm}$ from the surface of the filled tray to the top of the landmine. The sphere, dihedral and trihedral allow for GPR channel amplitude and phase correction.

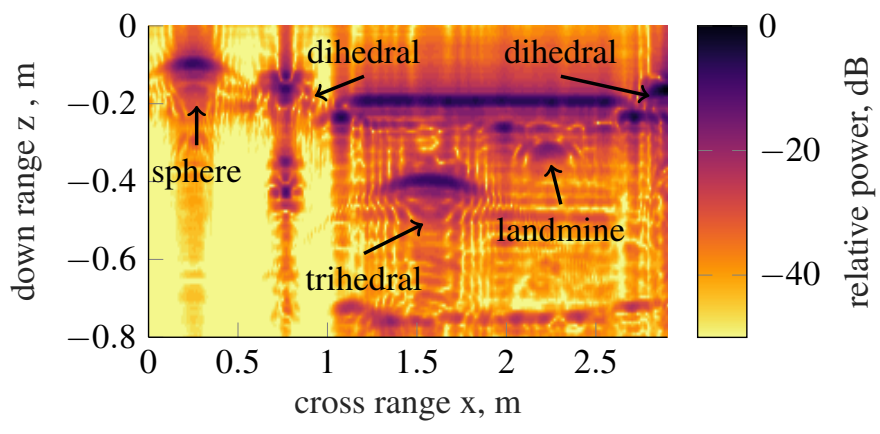

Figure 3. B-scan image of the radar scene with the surface and buried targets in the $\mathrm{HH}$ polarisation state i.e. H-horizontally polarized antenna used for transmission and H-horizontally polarised antenna used for reception.

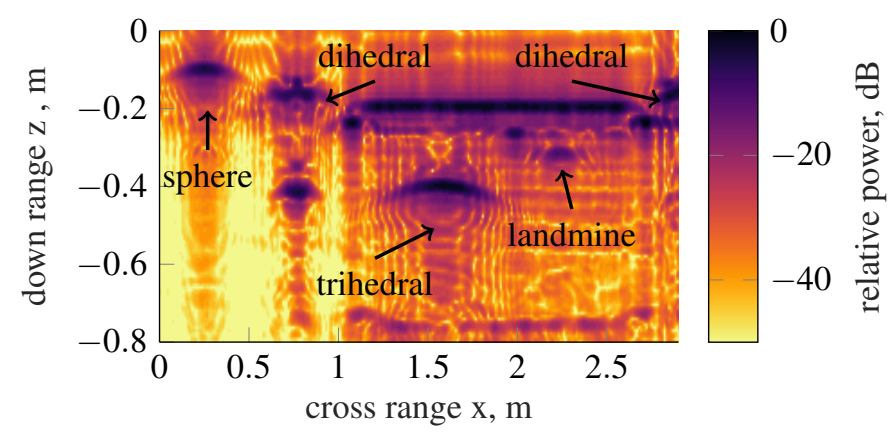

Figure 4. B-scan image of the radar scene with the surface and buried targets indicated in the VV polarisation state i.e. V-vertically polarized antennas used for transmission and reception.

\subsection{Signal Analysis}

The VNA uses a Stepped Frequency Continuous Waveform (SFCW) consisting of $N$ pulses, equally separated by $\Delta f$ Hertz. The phase of the sampled quadrature mixer output is,

$$
\phi_{n}=-2 \pi\left(f_{o}+n \Delta f\right) \frac{2 z}{c}
$$

where $z$ is the range to the object and $f_{o}$ is the frequency of the first transmitted pulse [12] and may be expressed as the complex spectral return $S$ of amplitude $A_{n}$ as, 


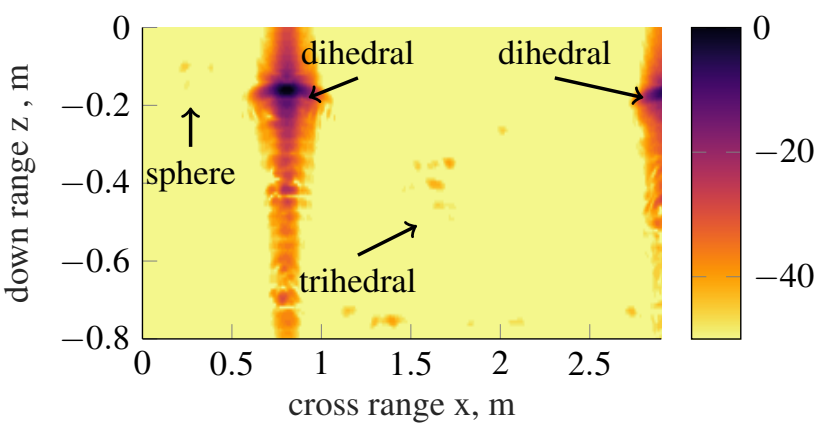

Figure 5. B-scan image of the radar scene with the surface and buried targets indicated in the $\mathrm{VH}$ polarisation state i.e. V-vertically polarized antenna used for transmission and $\mathrm{H}$-horizontally polarised antenna used for reception.

Table 1. Waveform specification for the $\mathrm{X}$-Band ground penetrating radar.

\begin{tabular}{ll} 
System Parameter & Specified Value \\
\hline pulse waveform & SFCW \\
RF power & $5 \mathrm{dBm}$ \\
Polarization & linear \\
bandwidth & $4 \mathrm{GHz}(8.2-12.4 \mathrm{GHz})$ \\
frequency steps & 1601 \\
frequency step & $2.5 \mathrm{MHz}$ \\
range to surface & $58 \mathrm{~cm}$ \\
\hline
\end{tabular}

$$
S\left(f_{n}\right)=A_{n} \exp \left(j \phi_{n}\right)
$$

The $N$ complex samples in each burst are Inverse Fast Fourier Transformed (IFFT) to a series of complex range reflectivity profiles. It can be shown that taking the IFFT (or FFT) of $M$ samples (from $N$ pulses) corresponding to a range bin divides the range bin into finer subdivisions resulting in a smoother range profile [13]. In this experiment a total of $N=801$ sample steps were taken in each measurement and resampled to $M=4096$. The antenna mutual coupling in Eand H-plane data is subtracted from the measurements of the buried objects to reduce antenna coupling artifacts in range profiles.

Figures 3-5 show the processed results for the range profiles (or B-scan imagery) for the experimental environment in the various polarisations collected. The sphere and trihedral as expected exhibit strong backscattering in $\mathrm{HH}$ and VV co-polarisated channels. The buried landmine (with no metal content) is also clearly visible in these polarisation states. The surface laid dihedrals also show strong co-polarised responses. This is because the dihedral is orientated at $22.5^{\circ}$ to the incident field and back scatters equally in both co-polarised states. The sphere and trihedrals as anticipated show weak cross-polarised response; however, the landmine in this case unfortunately also exhibits no response. In general, the subsurface imagery contains significant noise and clutter content potentially obsecuring subsurface objects. Thus techniques to improve the system performance and more fully exploit polarimetry to suppress clutter are sought.

\section{Channel Calibration}

Assuming the Back Scatter Alignment convention (BSA) and a Cartezian coordinate frame then the incident and scattered waves are characterized by two linear polarisations $\mathrm{H}$ horizontal and V-vertical (w.r.t electric field) and are defined according to [6]. Then the polarisation scattering process at the target can be represented by a scattering matrix $[S]$ for both the monostatic and bistatic case in terms of their Jones vector,

$$
\left[\begin{array}{c}
E_{H}^{s} \\
E_{V}^{s}
\end{array}\right]=\frac{e^{j k r}}{r}\left[\begin{array}{ll}
S_{H H} & S_{H V} \\
S_{V H} & S_{V V}
\end{array}\right] \cdot\left[\begin{array}{c}
E_{H}^{i} \\
E_{V}^{i}
\end{array}\right]
$$

where $E^{i}$ is the incident electric field and $E^{s}$ is the scattered electric field and matrix $[S]$ is the scattering matrix and the complex scattering coefficients are a function of bistatic angle. Since the diagonal elements relate the same polarization of incident and scattered fields, they are known as the co-polar terms, while the off-diagonal elements are known as crosspolar terms, as they relate the orthogonal polarization of the incident and scattered fields. In the case of a monostatic system, the reciprocity theorem results in equal cross-polar terms i.e. $S_{H V}=S_{V H}$. The $e^{j k r} / r$ term takes into account the propagation effects, both in amplitude and phase and holds only in the far-field zone. In practical laboratory applications with limited measurement range the validy must be checked.

Since, the receive and transmit antennas location are offset, the system has a small bistatic angle. The principles of radar polarimetry are derived for the monostatic case thus the bistatic geometry must be taken account off and a correction factor applied to the scattering matrix Eqn. (3).

The approach taken is to reduce the problem to a twodimensional problem and characterise the amplitude and phase variation, for all polarisations, for a small range of bistatic angles between the transmit and receive antennas. Figure 6 shows the calibration scheme, viewed from the top of the antennas. The transmit antenna stay static over the calibration object, in this case the dihedral, while the receive antenna is linearly offset over $N$ positions. The scattering measurements are taken in $1 \mathrm{~cm}$ increments over a $20 \mathrm{~cm}$ interval. The results for the deviation in phase for each polarization at each position are shown plotted in Figure 7. The phases can now be extrapolated to the zero receiver antenna position providing an estimate of phase in the monostatic case. It can be observed from the figure that the phase change is relatively small suggesting the antenna array configuration is quasi-monstatic. The average phase $(\bar{\psi})$ for all four polarisations at position zero was taken as the phase correction factor. Thus,

$$
S_{\text {bistatic }}=\left[\begin{array}{ll}
S_{H H} & S_{H V} \\
S_{V H} & S_{V V}
\end{array}\right]
$$

Now transforms the measurement to the monostatic case,

$$
S_{\text {monostatic }}=\left[\begin{array}{ll}
S_{H H} \overline{\psi_{H H}} & S_{H V} \overline{\psi_{H V}} \\
S_{V H} \overline{\psi_{V H}} & S_{V V} \overline{\psi_{V V}}
\end{array}\right]
$$

The polarimetric measurement data is then corrected for the monostatic case. 


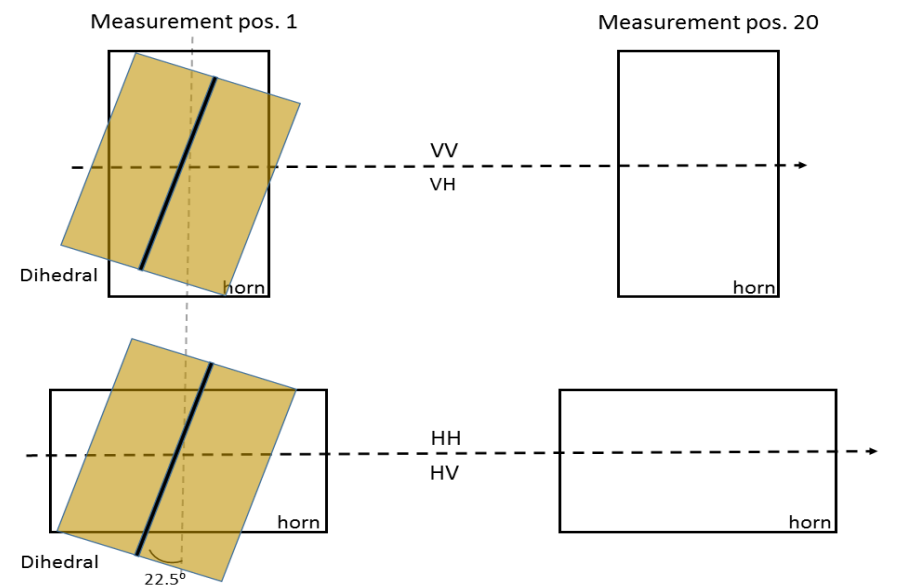

Figure 6. Schematic diagram illustrating the phase correction calibration procedure from above. The target is a Dihedral with seam orientated $22.5^{\circ}$ to the direction of movement. Two $T E_{01}$ mode horn antennas apertures are located side-by-side in $\mathrm{H}$ - or V-polarisations over the calibration target as indicated and the transmit antenna is fixed while the recieve antenna is moved in centimeter increments away.

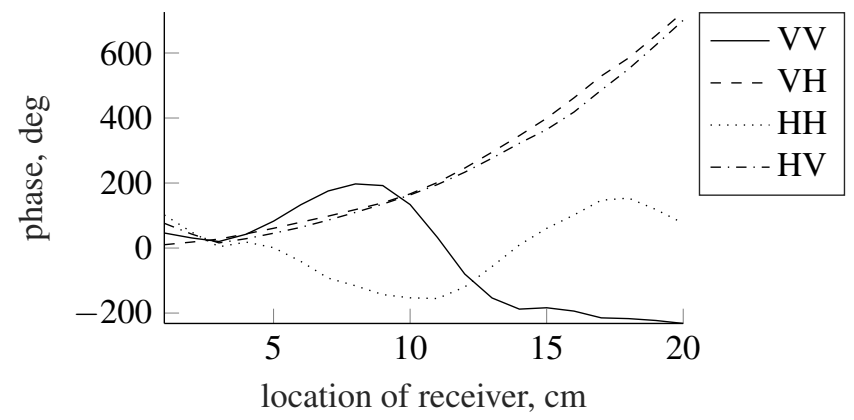

Figure 7. Measured phase $\psi$ for a $22.5^{\circ}$ inclinded seam dihedral target as a function of position and linear polarisation state.

\section{Yamaguchi Polarimetry Decomposition}

In [10] Moriyama demonstrates it is possible to synthesis a radar channel at any polarisation state from the scattering matrix. If $E_{t}$ is the transmitted wave and $E_{s}$ the scattered wave from the target then $E_{t}$ can be defined by the Jones matrix as,

$$
E_{t}=\frac{1}{\sqrt{1+\rho \rho^{*}}}\left[\begin{array}{l}
1 \\
\rho
\end{array}\right] .
$$

where $\rho$ is the polarisation ratio. The scattered wave is related to the transmitted wave via the scattering matrix where $S[H V]$ represents the targets polarimetric scattering characterisitics in the $H V$ basis. The co-polar and cross-polar powers are then obtain as follows; for the case of co-polar power,

$$
P_{c o}=\left|E_{t}^{T}(H V) \cdot S[H V] \cdot E_{t}(H V)\right|^{2}
$$

where $T$ denotes the transpose and $\perp$ denotes the orthogonal polarisation. The complex null polarisation value is calculated from the appropriate polarimetric co- and cross-polar power expression by setting it to zero and solving for the roots of the matrix as given by [10],

$$
\rho_{\text {co,null }}=\frac{S_{H V} \pm \sqrt{S_{H V}^{2}-S_{H H} \cdot S_{V V}}}{S_{V V}}
$$

and co-polar power is

$$
P_{c o}=\left|\frac{S_{H H}+2 \cdot S_{H V} \rho+S_{V V} \cdot \rho^{2}}{1+\rho \rho^{*}}\right|^{2}
$$

In the case of cross-polar power $E_{t}$ the transmitted field is again defined by the Jones scattering matrix,

$$
E_{\perp, t}=\frac{1}{\sqrt{1+\rho \rho^{*}}}\left[\begin{array}{c}
\rho^{*} \\
-1
\end{array}\right]
$$

and the cross-polar power is deduced as,

$$
P_{x}=\left|E_{\perp, t}^{T}(H V) \cdot S[H V] \cdot E_{t}(H V)\right|^{2}
$$

where the roots of the equation are again found by setting $P_{x}$ to zero

$$
\rho_{x, \text { null }}=\frac{-B \pm \sqrt{B^{2}-4 A C}}{2 A}
$$

where,

$$
\begin{gathered}
A=S_{H H}^{*} S_{H V}+S_{H V}^{*} S_{V V} \\
B=\left|S_{H H}\right|^{2}-\left|S_{V V}\right|^{2} \\
C=-A^{*} \\
P_{x}=\left|\frac{S_{H H} \rho^{*}+S_{H V}\left(\rho \rho^{*}-1\right)-S_{V V} \rho}{1+\rho \rho^{*}}\right|^{2}
\end{gathered}
$$

The polarimetric clutter matrix, is agnostic in the sense that it doesnt know the type of clutter we wish to filter e.g. it could be antenna coupling, or as here, subsurface clutter in the sand. Once the clutter region is selected the polarimetric pixels are extracted to form the clutter matrix, $S_{\text {sand }}$.

$$
S_{\text {sand }}=\left[\begin{array}{ll}
1.430+1.437 j & 0.150-0.150 j \\
0.150-0.150 j & 1.352-0.661 j
\end{array}\right] \cdot 10^{-2}
$$

where $\rho$ is the polarisation ratio and there are two possible roots to Equation (8) for the sand co-polar scattering matrix polarisation given below,

$$
\begin{aligned}
& \rho_{c o, 1}=-0.7133 \cdot 10^{-2}+0.4566 j \\
& \rho_{c o, 2}=-0.7133 \cdot 10^{-2}-0.4566 j .
\end{aligned}
$$

Using the value for $\rho$ in Equation (4.) the co-polar null polarisation power responses was calculated and is shown in Figure 8a. Similarly, the cross-polar null polarisation was calculated and the cross-polar power result processed and shown in Figure 8b. The B-scans imagery show reduced clutter levels in both co- and cross-polar null filters. The dihedral response has been strongly reinforced due to back scatter signal present 
in all polarisations while the sphere response has weakened mainly due to its lack of cross-polar signals. The trihedral and landmine are well contrasted more so in the co-polar than cross-polar power.

\section{Coherent Decomposition Parameters}

Coherent decomposition parameters that take account of polarisation were also invesigated for subsurface clutter reduction. These include Stokes $I_{0}$ which represents the total polarisation intensity of a wave and Pauli $a, b$ and $c$ parameters which decompose the $[S]$ matrix into various scattering mechansims. Where the $a$ scattering matrix corresponds single scattering of a plane surface (single or odd bounce scattering). The $b$ scattering is from a corner surface oriented at $0^{\circ}$ (double- or even-bounce scattering) since the polarization of the returned wave is mirrored respect to the one of the incident wave. The $c$ matrix scattering is from a corner surface oriented at $45^{\circ}$ (double- or even-bounce scattering). The decomposition parameters can be related to the $S[H V]$ basis and the Jones vector for plane polarised electromagnetic waves as,

$$
\begin{gathered}
I_{o}=\left|S_{H H}\right|^{2}+\left|S_{V V}\right|^{2} \\
a=\left(S_{H H}+S_{V V}\right) / \sqrt{2} \\
b=\left(S_{H H}-S_{V V}\right) / \sqrt{2} \\
c=\left(S_{H V}+S_{V H}\right) / \sqrt{2}
\end{gathered}
$$

The summation of linear polarisation intensities Stokes $I_{0}$ and the complex Pauli decompositions into the $a, b$ and $c$ scattering mechanisms are computed and shown in Figure 9.

\section{Discussion and Summary Comments}

An experimental set-up for performing subsurface measurements in full polarimetry has been described and microwave measurements for different surface laid and buried target and reference objects taken. A phase calibration technique was developed and applied to correct for small bistatic angle in the measurement setup restoring the system to monostatic basis. Both Yamaguchi and coherent polarimetric decompositions were investigated to provide clutter suppression of the subsurface.

The Yamaguchi technique provides co- and cross-polar image products with reduced clutter artifacts and enhanced image contrast. In these image products the dihedrals (with seam orientated $22.5^{\circ}$ to scan direction) provides a very strong backscatter response since they reflect equally in $\mathrm{HH}$ and VV polarisations. The buried landmine and trihedral are also clearly visible.

The Stokes $I_{O}$ intensity parameters provides a much reduced clutter image product; however, all the objects' intensities are also reduced to the point that the weak landmine response is undetectable. The Pauli $a$ and $b$ image products clearly show all targets with much reduced clutter. The undesired strong clutter echo present in the Yamaguchi null filter technique is now absent. Interestly, because the dihedrals were arranged at $22.5^{\circ}$ they are observed, as would be expected, to possess double or even bounce scattering features in the Pauli $c$ image product. In the Pauli $c$ decomposition the landmine and trihedral and sphere are not observable indicating an absence of double bounce polarisation features in the diagonal plane. Combining the information from these image products enables subsurface targets to be spatially localised, subsurface clutter suppressed and provides additional knowledge on target scattering mechanisms that could assist in there identification.

\section{Acknowledgment}

The authors thank the Find A Better Way charity for their support of this research under the DETERMINE programme (grant nuber 2015/001D).

\section{References}

[1] D.J. Daniels, "Ground Penetrating Radar", 2nd Edition, London, England: IET, (2007).

[2] H. Jing and T. Vladimirova,"Novel algorithm for landmine detection using C-scan ground penetrating radar signals",Seventh International Conference on Emerging Security Technologies (EST), Canterbury, , pp. 68-73, (2017).

[3] S. Sarjoghian and Y. Alfadhl and X. Chen,"Compact ultrawideband double-ridged horn antennas for medical imaging", Loughborough Antennas Propagation Conference, Burliegh Court, Loughborough, (2016).

[4] K. V. Hoel and S. Kristoffersen and J. Moen and G. Holm and T. S. Lande, "Characterization of a 3D printed wideband waveguide and horn antenna structure embedded in a UAV wing", 10th European Conference on Antennas and Propagation (EuCAP), (2016).

[5] Y. Yamaguchi, T.Nishikawa, M. Sengoku, W.-M. Boerner, H.J. EOM,"Fundamental Study on Synthetic Aperture FM-CW Radar Polarimetry",IEICE Transactions on Communications, vol. E77B no. 1, Jan. (1994).

[6] F.T. Ulaby, C. Elachi, "Radar Polarimetry for Geoscience Applications", Norwood, MA: Artech House, (1990).

[7] W. S. Benedix et al., "External calibration of GPR antenna accommodated on a rover", Proceedings of the Fourth European Conference on Antennas and Propagation (EuCAP), (2010).

[8] D. Kahny, K. Schmitt and W. Wiesbeck, "Calibration of Bistatic Polarimetric Radar Systems",IEEE Transactions on Geoscience and Remote Sensing, vol. 30, no. 5, pp. 847-852, (1992).

[9] K. Schmitt, D. Kahny and W. Wiesbeck, "Reduced Calibration Procedure for Multiple Bistatic RCS measurements",Geoscience and Remote Sensing Symposium, pp. 1201-1203, (1992).

[10] T. Moriyama, H. Kasahara, Y. Yamaguchi, H. Yamada, "Advanced Polarimetric Subsurface FM-CW Radar",IEEE Transactions on Geoscience and Remote Sensing, (1998).

[11] E. Banks, "Brassey's Essential Guide To Anti-Personnel Landmines, Recognizing and Disarming", Potomac Books Inc, 1st Edition, ISBN 1-85753-228-7, (1998).

[12] M. Skolnik, "Radar Handbook", McGraw-Hill, 2nd Edition ISBN 0-07-057913-X, (1990).

[13] D. R. Wehner,"High Resolution Radar", Artect House, 2nd Edition, Chap. 5, (1995). 


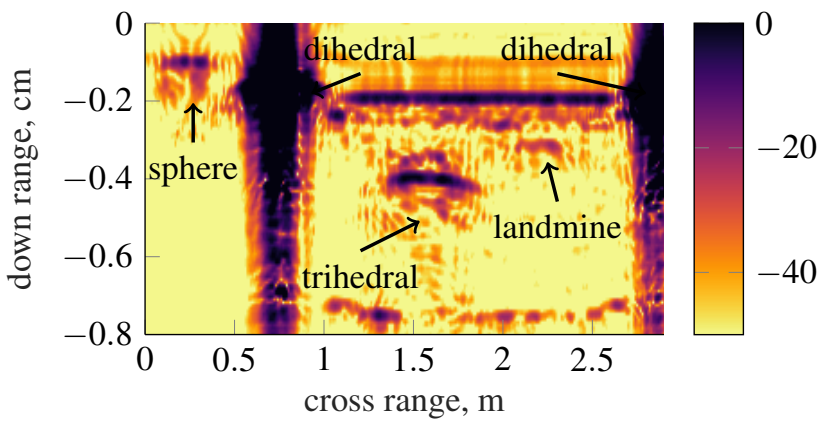

(a) Co-polar null polarisation power.

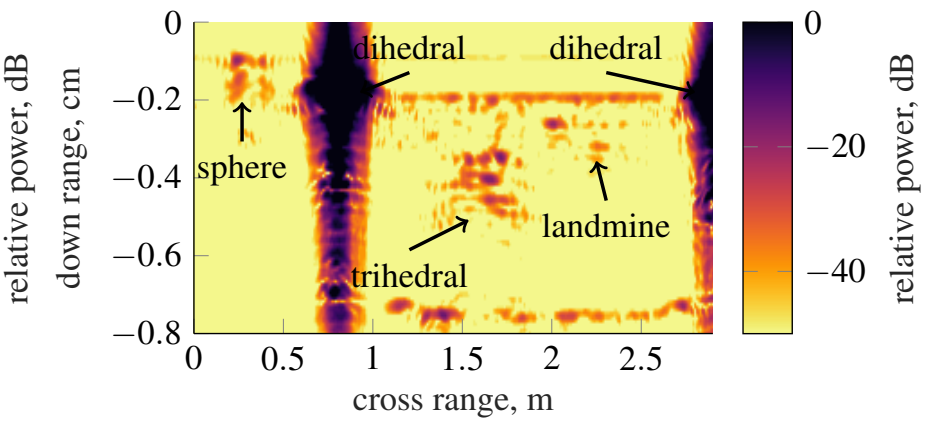

(b) Cross-polar null polarisation power.

Figure 8. Bscan images for the $P_{c o}$ co- and $P_{x}$ cross-polar null polarisation filtered powers (compare with Figs 3-5).

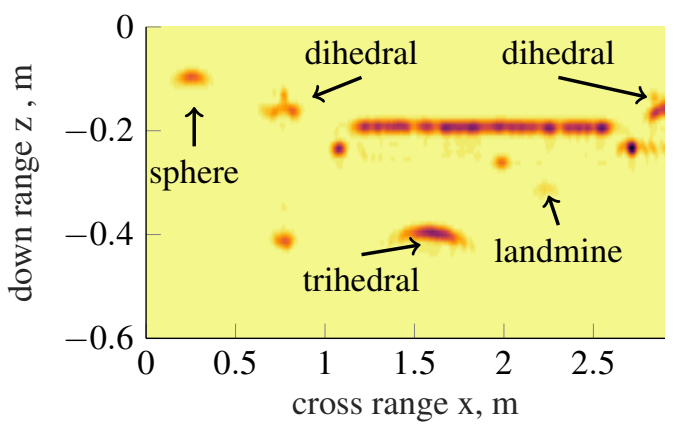

(a) Stokes $I_{0}$ parameter.

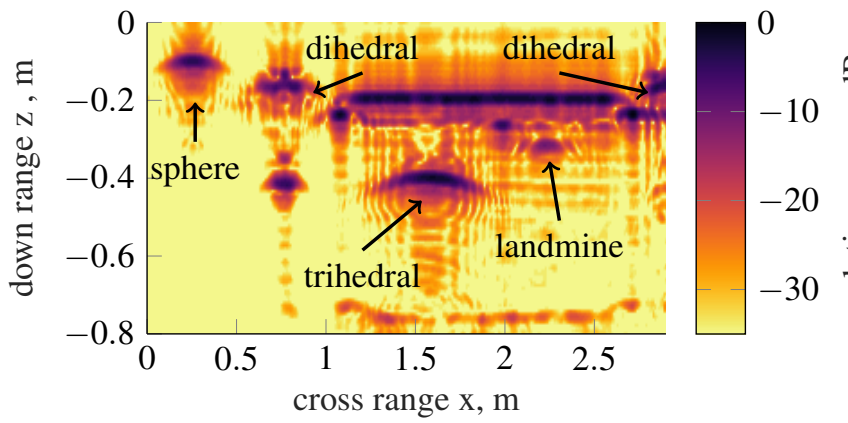

(c) Pauli $b$ decomposition parameter.
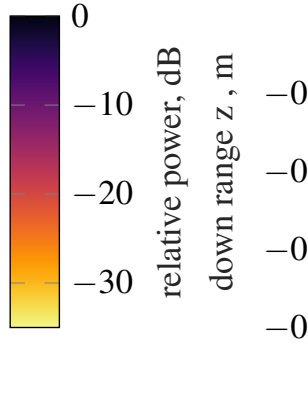

(b) Pauli $a$ decomposition parameter

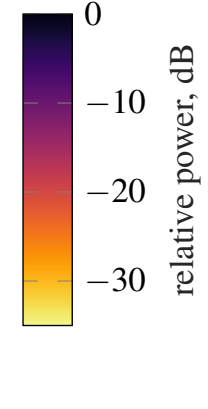

